\title{
Predicting Saturated Hydraulic Conductivity by Artificial Intelligence and Regression Models
}

\author{
R. Rezaei Arshad, ${ }^{1}$ Gh. Sayyad, ${ }^{1}$ M. Mosaddeghi, ${ }^{2}$ and B. Gharabaghi ${ }^{3}$ \\ ${ }^{1}$ Department of Soil Science, Faculty of Agrriculture, Shahid Chamran University of Ahvaz, Ahvaz, Iran \\ ${ }^{2}$ Department of Soil Science, College of Agriculture, Isfahan University of Technology, Isfahan, Iran \\ ${ }^{3}$ School of Engineering, University of Guelph, Guelph, ON, Canada
}

Correspondence should be addressed to R. Rezaei Arshad; rezae805@gmail.com

Received 7 April 2013; Accepted 12 May 2013

Academic Editors: G. Benckiser, D. Hui, H. K. Pant, and D. Zhou

Copyright (C) 2013 R. Rezaei Arshad et al. This is an open access article distributed under the Creative Commons Attribution License, which permits unrestricted use, distribution, and reproduction in any medium, provided the original work is properly cited.

\begin{abstract}
Saturated hydraulic conductivity $\left(K_{s}\right)$, among other soil hydraulic properties, is important and necessary in water and mass transport models and irrigation and drainage studies. Although this property can be measured directly, its measurement is difficult and very variable in space and time. Thus pedotransfer functions (PTFs) provide an alternative way to predict the $K_{s}$ from easily available soil data. This study was done to predict the $K_{s}$ in Khuzestan province, southwest Iran. Three Intelligence models including (radial basis function neural networks (RBFNN), multi layer perceptron neural networks (MLPNN)), adaptive neurofuzzy inference system (ANFIS) and multiple-linear regression (MLR) to predict the $K_{s}$ were used. Input variable included sand, silt, and clay percents and bulk density. The total of 175 soil samples was divided into two groups as 130 for the training and 45 for the testing of PTFs. The results indicated that ANFIS and RBFNN are effective methods for $K_{s}$ prediction and have better accuracy compared with the MLPNN and MLR models. The correlation between predicted and measured $K_{s}$ values using ANFIS was better than artificial neural network (ANN). Mean square error values for ANFIS, ANN, and MLR were 0.005, 0.02, and 0.17, respectively, which shows that ANFIS model is a powerful tool and has better performance than ANN and MLR in prediction of $K_{s}$.
\end{abstract}

\section{Introduction}

Soil hydraulic properties such as saturated hydraulic conductivity $\left(K_{s}\right)$ govern many soil hydrological processes; therefore, they are very important and even necessary in water and mass transport models and irrigation and drainage studies [1]. Direct measurement of soil hydraulic properties including $K_{s}$ is costly and time-consuming and becomes impractical due to spatial and temporal variabilities when hydrologic predictions are needed for large areas. Also it requires sophisticated measurement devices and skilled operators [2]. In the past few decades, as an alternative, indirect approximation of hydraulic properties from some basic and easily measured soil properties (such as clay, sand, and silt contents, and bulk density) using pedotransfer functions (PTFs) has received considerable acceptance [3-7]. "Pedotransfer function" was first introduced for empirical regression equations relating water and solute transport parameters to the basic soil properties that are available in soil survey [8].

The $K_{s}$ is an important soil hydraulic property often estimated using PTFs. Different methods such as regression models [3, 9-11] and artificial neural networks (ANN) are available for derivation of PTFs. In recent years, PTFs constructed by using artificial neural networks (ANN) (especially feed forward ANN) have proven popular with many researchers. ANN-PTFs have been developed by researchers such as Minasny et al., Minasny and McBratney, and Pachepsky et al. $[5,6,11]$. The overall conclusion made by these (and other) investigators was that when the number of input parameters is greater than three, ANN usually performs better than regression techniques, particularly when uncertainties in the quality of the data were small [12]. Multilayer perceptron (MLP) and radial basis function (RBF) are two of the most widely used neural network architecture. General 
difference between MLP and RBF is that RBF is a localist type of learning which is responsive only to a limited section of input space. RBF utilizes a local learning strategy versus MLP global learning and this leads to a higher rate of accuracy and faster training of RBF [13].

Adaptive neuro-fuzzy inference system (ANFIS) can be applied as a further alternative technique [14]. Similar to ANN, the neuro-fuzzy system constructs input-output membership function relationships. The fuzzy system is set to learn from the training data by adjusting the parameters of the membership functions using the least-squares method. The trained system can be used as a PTF to predict properties of an unknown soil sample [11]. A close spatial relationship between soil hydraulic properties and other easily available soil and terrain attributes, as auxiliary variables, can be exploited to predict these hydraulic characteristics, as target variables, with a reasonable accuracy at unobserved locations [15].

State of the art shows that, in most previous studies, $K_{s}$ was predicted using regression and ANN models $[3,16,17]$. Many comparisons of PTFs have been made with respect to different data sets, different mathematical procedures (regression versus ANN models), and different input parameters. However, there are few studies which have used ANFIS model for developing PTFs. Wieland and Mirschel compared a feed-forward neural network (NN), a radial basis function network (RBF), and a trained fuzzy algorithm for regional yield estimation of agricultural crops (winter rye, winter barley) [18]. As mentioned before, ANFIS model has more ability to develop PTFs than ANN and regression models. Moreover unlike the tradition, radial basis function (RBF) technique, as far as our knowledge goes, has not been used for this comparison purpose. Therefore the objectives of this study were (a) to employ the techniques of ANN (MLR and RBF methods), ANFIS, and multiple linear regression (MLR) to predict saturated hydraulic conductivity using easily available soil properties, (b) to evaluate the performance of these techniques and determine the best methodology for prediction of saturated hydraulic conductivity.

An ANN is a highly interconnected network of many simple processing units called neurons, which are analogous to the biological neurons in the human brain. Neurons having similar characteristics in an ANN are arranged in groups called layers. The neurons in one layer are connected to those in the adjacent layers but not to those in the same layer. The strength of connection between the two neurons in adjacent layers is represented by what is known as connection strength or weight. An ANN normally consists of three layers, an input layer, a hidden layer, and an output layer. In a feedforward network, the weighted connections feed activations only in the forward direction from an input layer to the output layer. On the other hand, in a recurrent network additional weighted connections are used to feed previous activations back into the network. The structure of a feed-forward ANN is shown in Figure 1 [19].

In Figure 1, the circles represent neurons; the lines joining the neurons represent weights; sand, silt, clay, and $\rho_{b}$ are input variables; $K_{s}$ represents the output variables; $w_{k j}$ and $W_{i k}$ represent the weights between input and hidden and

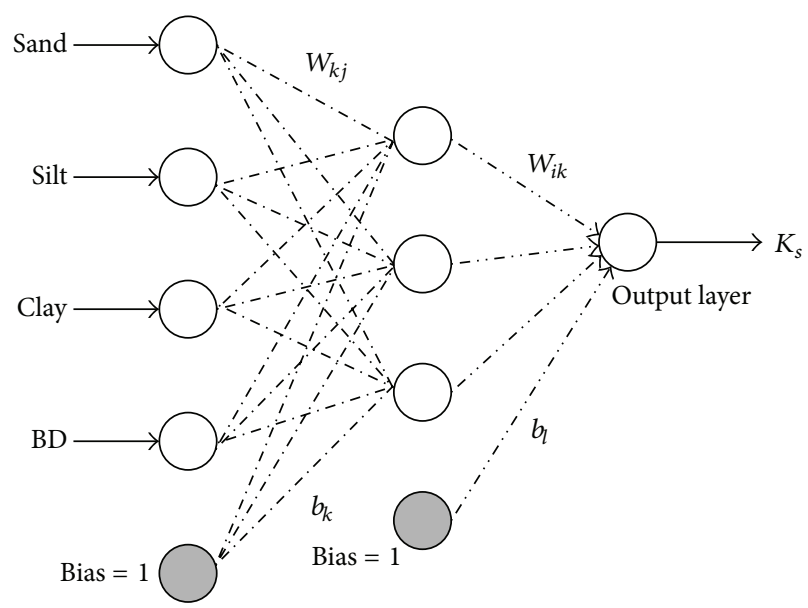

Figure 1: Structure of a feed-forward ANN.

hidden and output layers, respectively. Parameters $b_{k}$ and $b_{l}$ represent the bias of the corresponding hidden and output layer neurons. The role of bias in a neuron is to displace the original functional domain by a magnitude equal to that of the bias and thereby translate the area of influence to its activation state [19].

An important step in developing an ANN model is training of its weight matrix. The weights are initialized randomly in a suitable range and then updated using certain training mechanism. There are primarily two types of training mechanisms: supervised and unsupervised. A supervised training algorithm requires an external teacher to guide the training process. This typically involves a large number of examples (or patterns) of inputs and outputs for training. The inputs in an ANN are the cause variables and outputs are the effect variables of the physical system being modeled. The primary goal of training is to minimize the objective (error) function by searching for a set of connection strengths that cause the ANN to produce outputs that are equal to or closer to target data [19].

Multilayer perceptron (MLP) and radial basis function (RBF) are two of the most widely used neural network architecture in literature for classification or regression problems. They are robust classifiers with the ability to generalize for imprecise input data. Both artificial neural network and fuzzy logic are used in ANFIS's architecture. ANFIS is consisted of if-then rules and couples of input-output. ANFIS training uses learning algorithms of neural network [14, 20, 21]. An adaptive network, as its name implies, is a network structure consisting of nodes and directional links through which the nodes are connected. Moreover, parts or all of the nodes are adaptive, which means each output of these nodes depends on the parameters pertaining to this node and the learning rule specifies how these parameters should be changed to minimize a prescribed error measure [14]. ANFIS is a multilayer feed-forward network where each node performs a particular function on incoming signals. Both square and circle node symbols are used to represent different properties of adaptive learning (Figure 2). To perform desired input-output characteristics, adaptive learning parameters 


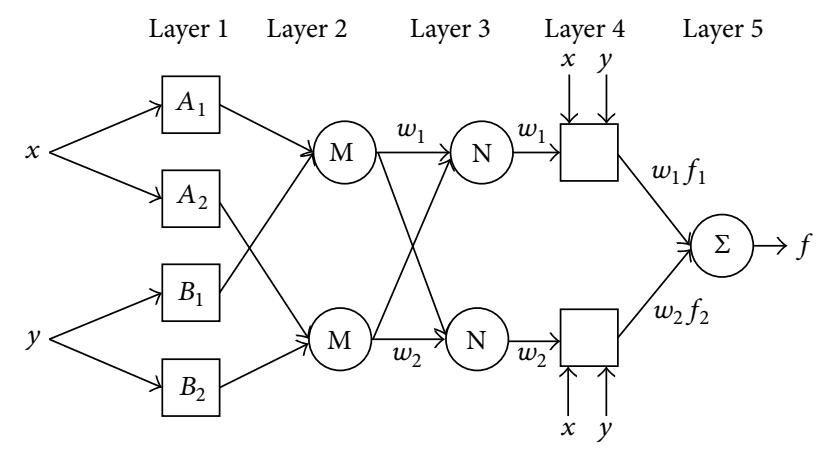

FIGURE 2: ANFIS architecture.

are updated based on gradient learning rules [14, 22, 23]. For simplicity, we assume that the fuzzy inference system under consideration has two inputs, $x$ and $y$, and one output $z$. suppose that the rule base contains two fuzzy if-then rules of Takagi and Sugeno's type.

Rule 1. If $x$ is $A_{1}$ and $y$ is $B_{1}$ then $f_{1}=p_{1} x+q_{1} y+r_{1}$.

Rule 2. If $x$ is $A_{2}$ and $y$ is $B_{2}$ then $f_{2}=p_{2} x+q_{2} y+r_{2}$,

where $A_{i}$ and $B_{i}$ are the fuzzy sets, $f_{i}$ are the outputs within the fuzzy region specified by the fuzzy rule, and $p_{i}, q_{i}$, and $r_{i}$ are the design parameters that are determined during the training process. The ANFIS architecture to implement these two rules is shown in Figure 2, in which a circle indicates a fixed node, whereas a square indicates an adaptive node.

Layer 1. Every node $i$ in this layer is a square node with a node function:

$$
\begin{array}{cc}
o_{i}^{1}=\mu A_{i}(x), & i=1,2 \\
o_{i}^{1}=\mu B_{i-2}(y), & i=3,4,
\end{array}
$$

where $x$ is the input to node $i$, and $A_{i}$ is the linguistic label (small, large, etc.) associated with this node function, and where $\mu A_{i}(x), \mu B_{i-2}(y)$ can adopt any fuzzy membership function. Usually, we choose $\mu A_{i}(x)$ and $\mu B_{i-2}(y)$ to be bellshaped with a maximum equal to 1 and a minimum equal to 0 , such as

$$
\mu A_{i}(x)=\frac{1}{1+\left\{\left(\left(x-c_{i}\right) / a_{i}\right)^{2}\right\}^{b}},
$$

where $\left(a_{i}, b_{i}\right.$, and $\left.c_{i}\right)$ is the parameter set. Parameters in this layer are referred to as premise parameters.

Layer 2. The nodes in this layer are fixed. These are labeled as $\mathrm{M}$ to indicate that they play the role of a simple multiplier. The outputs of these nodes are given by

$$
o_{i}^{2}=w_{i}=\mu A_{i}(x) \mu B_{i}(y), \quad i=1,2,
$$

which are the so-called firing strengths of the rules.
Layer 3. Every node in this layer is a circle node labeled as N. The $i$ th node calculates the ratio of the $i$ th rule's firing strength to the sum of all rules' firing strengths:

$$
o_{i}^{3}=\bar{w}=\frac{w_{i}}{w_{1}+w_{2}}, \quad i=1,2 .
$$

For convenience, outputs of this layer will be called normalized firing strengths.

Layer 4. In this layer, the nodes are adaptive nodes. The output of each node in this layer is simply the product of the normalized firing strength and a first-order polynomial (for a first-order Sugeno model). Thus, the outputs of this layer are given by

$$
o_{i}^{4}=\bar{w}_{i} f_{i}=w_{i}\left(p_{i} x+q_{i} y+r_{i}\right), \quad i=1,2 .
$$

Parameters in this layer will be referred to as consequent parameters.

Layer 5. The single node in this layer is circle node labeled as $\mathrm{S}$ that computes the overall output as the summation of all incoming signals; that is,

$$
o_{1}^{5}=\sum_{i=1}^{2} \bar{w}_{i} f_{i}=\frac{\sum_{i=1}^{2} w_{i} f_{i}}{w_{1}+w_{2}} .
$$

It is seen that there are two adaptive layers in this ANFIS architecture, namely, the first layer and the fourth layer. In the first layer, there are three modifiable parameters $\left\{a_{i}, b_{i}, c_{i}\right\}$, which are related to the input membership functions. These parameters are the so-called premise parameters. In the fourth layer, there are also three modifiable parameters $\left\{p_{i}, q_{i}, r_{i}\right\}$, pertaining to the first-order polynomial. These parameters are the so-called consequent parameters [14].

\section{Materials and Methods}

2.1. Data Collection. The data used in this study were obtained from the reports of basic soil science and land reclamation studies conducted by Khuzestan Water and Power Organization. Khuzestan province is located in the southwest of Iran from east $47^{\circ} 40^{\prime}$ to $50^{\circ} 33^{\prime}$ longitude and north of latitude. Khuzestan province has mild winters and very hot summers [24].

The data set which was used to develop the PTFs included the data from 175 soil profiles from different parts of Khuzestan province. Soil samples were taken from the $0-30 \mathrm{~cm}$ layer. The data consisted of five soil properties (four as independent variables and one as dependent variable). Independent variables were the percentages of clay $(\mathrm{C})$, silt $(\mathrm{Si})$, sand $(\mathrm{S})$, and bulk density $\left(\rho_{b}\right)$. Saturated hydraulic conductivity $\left(K_{s}\right)$ was considered as dependent (output) variable. Soil samples were air-dried and sieved by a $2 \mathrm{~mm}$ sieve for physical analysis. Particle size distribution was determined using pipet method. $\rho_{b}$ was determined on undisturbed samples using cylinder method, being made of $10 \mathrm{~cm}$ and $100 \mathrm{~cm}^{3}$ cylinders, after drying $24 \mathrm{~h}$ in $105^{\circ} \mathrm{C}$ ovens. Saturated hydraulic conductivity 
was measured using inversed auger hole method. The principle of the auger hole test above the water table consists of boring a hole to given depth, filling it with water and measuring the rate of fall of the water level [25].

MLP, RBF, ANFIS and MLR of pedotransfer functions were used to predict the $K_{s}$. The data set was divided into two separate data sets: the training one (80\%) and the testing one $(20 \%)$. The training data set was used to train the ANN, ANFIS, and MLR models, whereas the testing data set was used to verify the accuracy and the effectiveness of the trained models. A computer program was run in MATLAB (ver.7.6.0.323) to train and to test the data set using ANFIS and ANN structures for $K_{s}$ prediction. The performances of new techniques (ANN and ANFIS) were compared with MLR method.

2.2. Regression Analysis. In the multiple-linear regression (MLR) analysis, first, the most essential input variables were selected using backwards stepwise method, and then linear, quadratic, and possible interaction terms of these basic soil properties were investigated using the SPSS software. The general form of the regression equations was:

$$
Y=a+b_{1} X_{1}+b_{2} X_{2}+\cdots+b_{i} X_{i},
$$

where $Y$ is the dependent variable representing $K_{s}, a$ is the intercept, $b_{1}, \ldots, b_{i}$ are regression coefficients, and $X_{1}-X_{i}$ are independent variables referring to basic soil properties.

The same data sets were used in the derivation $(N=$ $130)$ and testing $(N=45)$ of PTFs developed using ANN, ANFIS, and regression methods for reliable comparison. The resulting functions were tested using data sets not included in the derivation procedure.

\subsection{Developing PTFs Using Artificial Neural Network Model} $(A N N)$. First step in developing an ANN model involves identifying input and output variables and normalizing the data between 0 and 1 . The method of channelized normalization was used to normalize the input and output data, wherein the data representing separate physical variables/parameters are normalized separately. Then the best ANN architecture is determined by finding the optimal number of hidden neurons through training of the various architectures using a trial and error method. Once the best ANN architecture is trained, it is validated using the testing data set.

In this study, two different types of ANN were developed. The first ANN model is multilayer perceptron (MLP) which is the most commonly used neural network structure in ecological modeling and soil science [26], whereas the second ANN model is radial basis function (RBF).

2.4. Neural-Fuzzy Model Design. For designing the neuralfuzzy system, the feed-forward multilayer neural networks with hybrid training algorithm and Takagi-Sugeno fuzzy inference system were used. In this model, the "gaussmf" membership function was used for input function, the "linear" membership function was used for output function, and defuzzification operation was performed by weight average function.
2.5. Performance Evaluation Criteria. Two standard statistics were used as evaluation criteria to evaluate the performance of ANN models (MLP and RBF) and ANFIS methods. These were mean square errors (MSE) and coefficient of determination $\left(R^{2}\right)$ as calculated using the following equations:

$$
\begin{gathered}
R^{2}=1-\frac{\sum_{i=1}^{N}\left(y_{i}-\widehat{y}_{i}\right)}{\sum_{i=1}^{N}\left(y_{i}-\bar{y}_{i}\right)^{2}}, \\
\mathrm{MSE}=\frac{1}{N} \sum_{i=1}^{N}\left(y_{i}-\bar{y}_{i}\right)^{2},
\end{gathered}
$$

where $N$ is the number of data set, $y_{i}$ is the measured value of output variable, $\hat{y}_{i}$ is the predicted value of output variable, and $\bar{y}_{i}$ is the average of predicted value of output variable.

\section{Results and Discussion}

Before developing PTFs using ANN, ANFIS, and MLR models, descriptive statistics of data were derived using SPSS (version 16). Descriptive statistics for the soil physical and hydraulic parameters which were used in the development (train) and validation (test) of PTFs using models are summarized in Table 1.

The studied soils have wide ranges of physical properties; for instance, the ranges of sand, silt, and clay contents, and $\rho_{b}$ are $4-70,12-66,9.4-62 \%$, and $0.96-1.77 \mathrm{Mg} \mathrm{m}^{-3}$ for training or derivation data set, respectively. Testing or validation data set had similar ranges. The studied soils are mostly originated from alluvial processes which probably cause such wide range in soil physical properties. The soils are on average with medium textures, where the mean silt percent and $\rho_{b}$ are 44.6 and $1.45 \mathrm{Mg} \mathrm{m}^{-3}$, respectively.

The $K_{s}$ values of the studied soils are mostly very high, ranging from 6.4-207 (cm day ${ }^{-1}$ ), with standard deviation of 29.4 and $10.7 \mathrm{~cm} \mathrm{day}^{-1}$ for training and testing data sets, respectively. A reason for high values of $K_{s}$ might be the large area of the studied region. Good structure and existence of macropores due to wide ranges of particle size distribution might be also responsible for high values of $K_{s}$.

3.1. PTFs Development Using MLR Model. In the regression analysis, normalizing the data distribution is one of the primary assumptions that have to be carried out. Therefore, the normality of the data was evaluated using the KolmogrovSmirnov method. $K_{s}, \rho_{b}$, sand, silt and clay data did not conform to normal distribution and were normalized using the natural-based logarithmic transformation. After normalizing data, in order to develop PTFs for predicting $K_{s}$ through MLR model, first the most essential input variables were selected using stepwise method, and then linear interaction terms of these basic soil properties were investigated by means of SPSS 16 software.

After training the regression model with training data set, the derived regression equation was as follows:

$$
\begin{aligned}
\operatorname{Ln} K_{s}= & 14.66-0.44 * \text { Ln sand }-1.37 * \text { Ln silt } \\
& -1.25 * \text { Ln clay }-2.8 * \operatorname{Ln} \rho_{b} .
\end{aligned}
$$


TABLE 1: Descriptive statistics of the datasets used for training and testing (ANN, ANFIS, and MLR).

\begin{tabular}{|c|c|c|c|c|c|c|c|c|c|}
\hline \multirow{2}{*}{ Variable } & \multirow{2}{*}{ Units } & \multicolumn{4}{|c|}{ Training data $(N=130)$} & \multicolumn{4}{|c|}{ Testing data $(N=45)$} \\
\hline & & Max. & Min. & Mean & S.D. & Max. & Min. & Mean & S.D. \\
\hline$\rho_{b}$ & $\mathrm{Mg} \mathrm{m}^{-3}$ & 1.77 & 0.96 & 1.45 & 0.14 & 1.74 & 1.20 & 1.45 & 0.13 \\
\hline Sand & $\%$ & 70.0 & 4.0 & 27.9 & 13.8 & 52.0 & 4.0 & 20.4 & 11.2 \\
\hline Silt & $\%$ & 66.0 & 12.0 & 44.6 & 10.6 & 78.0 & 18.0 & 47.5 & 10.8 \\
\hline Clay & $\%$ & 62.0 & 9.4 & 28.5 & 11.2 & 62.0 & 10.5 & 32.1 & 12.6 \\
\hline$K_{s}$ & $\mathrm{~cm} \mathrm{day}^{-1}$ & 207.0 & 6.9 & 27.4 & 29.4 & 60.0 & 6.4 & 18.2 & 10.7 \\
\hline
\end{tabular}

TABLE 2: Summary of statistical analyses of training and testing the MLR for $K_{s}$ prediction.

\begin{tabular}{lcccc}
\hline & \multicolumn{2}{c}{ Training } & \multicolumn{2}{c}{ Testing } \\
Model & $R^{2}$ & MSE & $R^{2}$ & MSE \\
\hline MLR & 0.69 & 0.01 & 0.5 & 0.17 \\
\hline
\end{tabular}

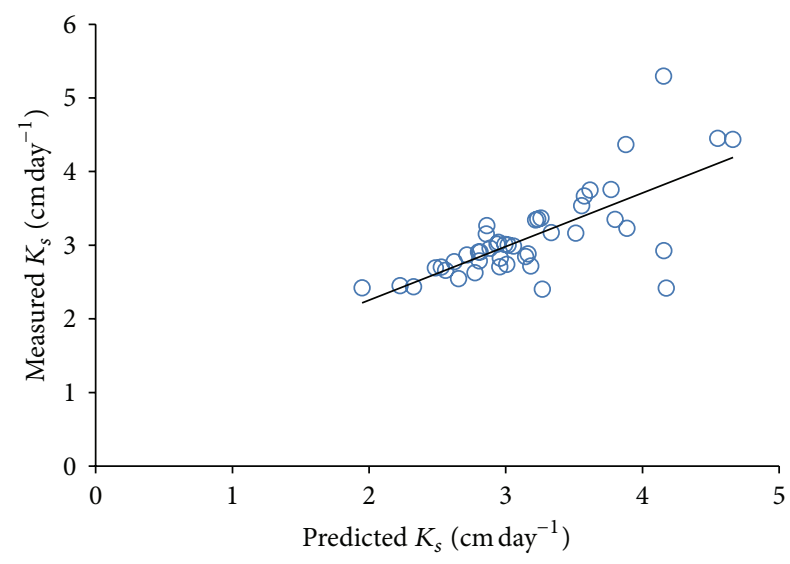

FIGURE 3: Measured versus predicted $K_{s}$ values using MLR.

In derived equation clay, sand, silt, and bulk density were chosen as the independent variables. After determining regression equation, the accuracy of MLR model was evaluated through comparing its predicted $K_{s}$ with experimental data. The obtained values of $R^{2}$ and MSE using MLR model for predicting $K_{s}$ are tabulated in Table 2. As observed from this table for test data set, the $R^{2}$ and MSE values that have been obtained are 0.5 and 0.17 , respectively. Merdun et al. obtained higher $R^{2}$ and RMSE values varied from 0.637 to 0.979 and from 0.013 to 0.938 for regression method, respectively [3]. The scatter plot of the measured against predicted $K_{s}$ values obtained from the MLR model for the test data set with a poor correlation coefficient is illustrated in Figure 3.

3.2. PTFs Development Using ANN Models. In current study two different algorithms of ANNs including radial basis function (RBF) and multilayer perceptron (MLP) models were investigated to predict $K_{s}$ through employing the same data set which was used by MLR. In order to reach this end, all data sets were first normalized between 0 and 1 to achieve effective network training. Luk et al. stated that neural networks trained on normalized data achieve better performance and faster convergence in general, although the advantages diminish as network and sample size become large [27]. Normalizing the data set was done through

$$
X_{\text {norm }}=0.5\left(\frac{X_{0}-\bar{X}}{X_{\max }-X_{\min }}\right),
$$

where $X_{\text {norm }}$ is the normalized value, $X_{0}$ is the actual value, $\bar{X}$ is average value, for each parameter, $X_{\max }$ is the maximum value and $X_{\min }$ is the minimum value. A three-layered feedforward ANN architecture with an input layer, one hidden layer, and an output layer was developed for predicting $K_{s}$ by means of both ANN models.

3.3. MLP Network. Table 3 shows the results of statistical analyses between the observed and MLP ANN-simulated values of $K_{s}$ for training and testing stages. For MLP network, the best architecture consists of four neurons in the input layer, seven neurons in the hidden layer, and one neuron in the output layer with Tansig and Purelin threshold functions for hidden and output layers, respectively, gave the best results. The $R^{2}$ and MSE values among the observed and predicted $K_{s}$ are 0.66 and 0.02 , respectively. The values are in accordance with the previous studies using ANN methods. Agyare et al. while estimating $K_{s}$ obtained $R^{2}$ and NMSE about 0.6 and 0.42 , respectively [26]. Merdun et al. obtained $R^{2}$ ranges and RMSE varied from 0.444 to 0.952 and from 0.020 to 3.511 , respectively [3]. Figure 4 shows the relationship between the measured and predicted $K_{s}$ values for testing stage indicating that MLP network can predict $K_{s}$ with acceptable accuracy. In order to employ RBF, Gaussian function that is the most widely used in applications was chosen as a threshold function for hidden layer. In the next step a regression analysis of the network response between ANN outputs and the corresponding targets was performed. Table 3 tabulated that the provided predictions between ANNs outputs and the corresponding targets using RBF model $\left(R^{2}=0.68, \mathrm{MSE}=0.02\right)$ and MLP were approximately similar and differences were not significant.

The levels of $R^{2}$ and MSE derived by both ANN models had higher accuracy than those derived by multiple linear regressions for predicting $K_{s}$ which was a support for those previous studies conducted by Merdun et al., Tamari et al., Yilmaz et al., and other researchers $[3,28,29]$. This is due to that, unlike the traditional regression PTFs ANNs do not require a priori regression model which relates input and output data that in general is difficult because these 
TABLE 3: Summary of statistical analyses of training and testing the MLP and RBF neural network for $K_{s}$ prediction.

\begin{tabular}{lccccrrr}
\hline \multirow{2}{*}{ Network } & \multirow{2}{*}{ Architecture } & \multirow{2}{*}{ Threshold function } & \multirow{2}{*}{ Spread } & \multicolumn{2}{c}{ Training } & \multicolumn{2}{c}{ Testing } \\
& & & & MSE & $R^{2}$ & MSE \\
\hline MLP & $4-7-1$ & Tansig-Purelin & & 0.88 & 0.007 & 0.66 \\
RBF & & Radbas & 45 & 0.9 & 0.005 & 0.68 \\
\hline
\end{tabular}

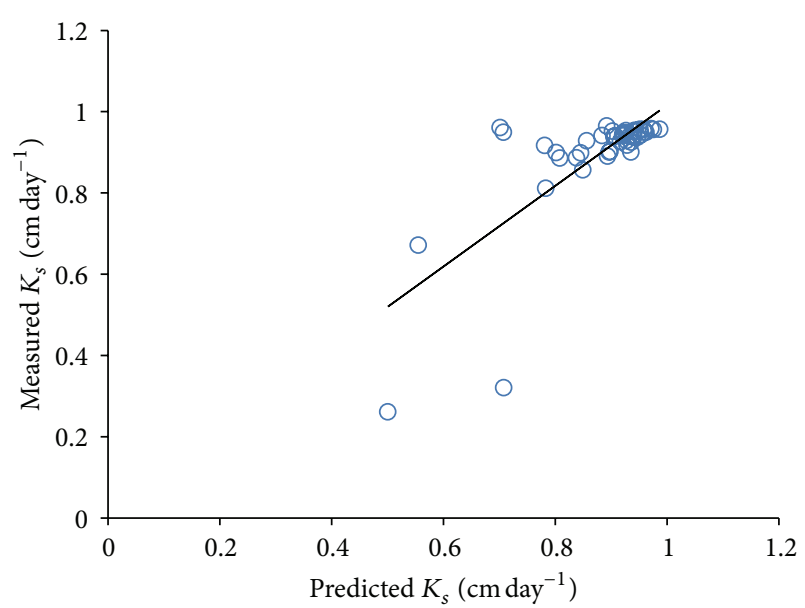

FIgURE 4: Measured versus predicted $K_{s}$ values using MLP network.

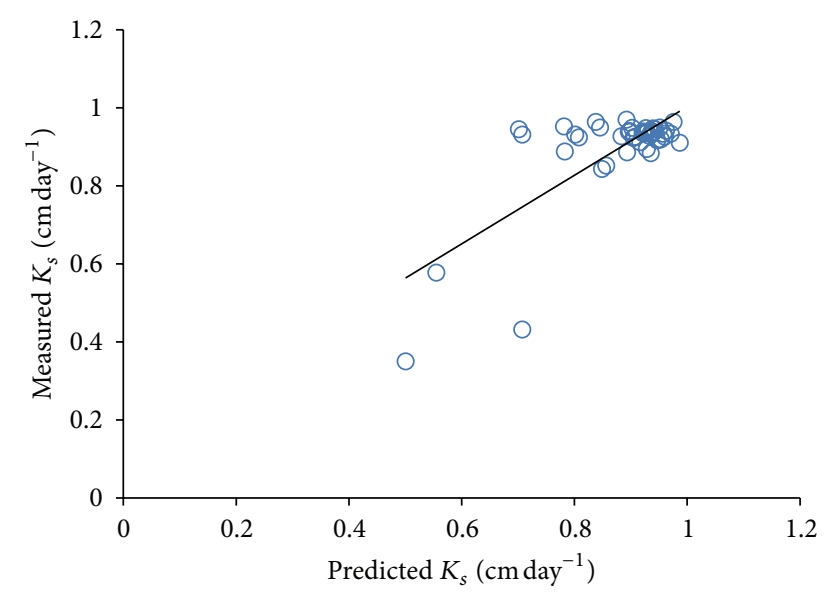

FIGURE 5: Measured versus predicted $K_{s}$ values using RBF network.

models are not known [30]. Also Minasny and McBratney, Pachepsky et al., and Tamari et al. stated that when the number of input parameters is greater than three, ANNs usually perform better than regression techniques, particularly when uncertainties in the quality of the data were small [11, 28, 31]. Additionally many investigations have indicated that a neural network with one hidden layer is capable of approximating any finite nonlinear function with very high accuracy [32, 33]. The scatter plots between measured and predicted $K_{s}$ using MLP and RBF models for testing stage with acceptable accuracy are indicated in Figures 4 and 5, respectively.

3.4. PTFS Development Using ANFIS Model. In order to develop PTFs for prediction $K_{s}$ through ANFIS model the
TABLE 4: Summary of statistical analyses of training and testing the ANFIS model for $K_{s}$ prediction.

\begin{tabular}{lcccc}
\hline & Training & & \multicolumn{3}{c}{ Testing } \\
$R^{2}$ & MSE & $R^{2}$ & MSE \\
\hline 0.72 & 0.009 & 0.71 & 0.005 \\
\hline
\end{tabular}

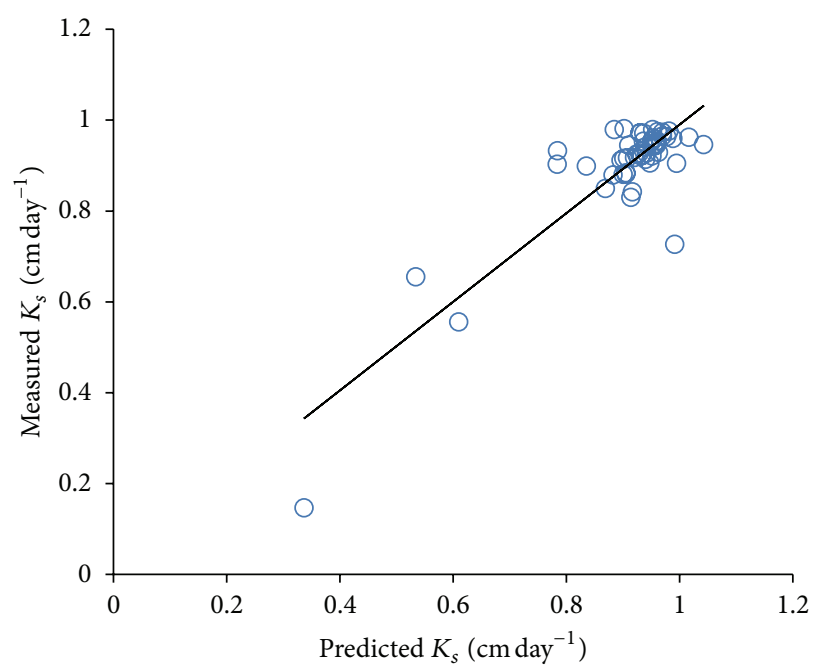

FIGURE 6: Results of running the ANFIS network in testing stage (actual versus predicted $K_{s}$ values).

same normalized data which employed by both ANN models were used as the input variables. In the ANFIS system, each input parameter might be clustered into several class values in layer 1 to build up fuzzy rules, and each fuzzy rule would be constructed using two or more membership functions in layer 2. Several methods have been proposed to classify the input data and to make the rules, among which the most widespread of them are grid partition and subtractive fuzzy clustering [34-36]. In this study subtractive fuzzy clustering was taken into consideration to develop the rule base relationship between the input and output variables. Sugeno fuzzy model, also known as Takagi-SugenoKang (TSK) model [37], that is, one of the most widespread methodologies for developing fuzzy rules was used in the current study to predict soil $K_{s}$.

The performance of the ANFIS model for the test data set and the related statistical evolutionary results are given in Figure 6 and Table 4, respectively. The values of 0.71 and 0.005 for $R^{2}$ and MSE parameters, respectively, for ANFIS testing stage, show higher prediction accuracy of ANFIS model than the ANN models. 


\section{Conclusions}

Predicting the saturated hydraulic conductivity $\left(K_{s}\right)$ of soil is one of the important subjects in modeling water flow and solute transport processes in the vadose zone. This paper presents the development and validation of PTFs for prediction of $K_{s}$ from basic soil properties by using ANN and ANFIS models in Khuzestan province, southwest Iran. The predictive capabilities of these methods were also compared using some evaluation criteria of testing data. The results revealed that the ANFIS model had superiority to the ANN models for $K_{s}$ prediction. Both applied ANNs algorithms exhibited acceptable accuracy. The comparison of the RBF and MLP networks indicates that they had approximately similar performance for prediction $K_{s}$ as the obtained results using RBF were slightly better than MLP ones, but their differences were not found significant. Among the employed ANN models, RBF prediction was more accurate than MLP network prediction.

\section{References}

[1] V. Bagarello, S. Sferlazza, and A. Sgroi, "Comparing two methods of analysis of single-ring infiltrometer data for a sandy-loam soil," Geoderma, vol. 149, no. 3-4, pp. 415-420, 2009.

[2] N. Islam, W. W. Wallender, J. P. Mitchell, S. Wicks, and R. E. Howitt, "Performance evaluation of methods for the estimation of soil hydraulic parameters and their suitability in a hydrologic model," Geoderma, vol. 134, no. 1-2, pp. 135-151, 2006.

[3] H. Merdun, Ö. Çinar, R. Meral, and M. Apan, "Comparison of artificial neural network and regression pedotransfer functions for prediction of soil water retention and saturated hydraulic conductivity," Soil and Tillage Research, vol. 90, no. 1-2, pp. 108116, 2006.

[4] J. Tomasella, Y. Pachepsky, S. Crestana, and W. J. Rawls, "Comparison of two techniques to develop pedotransfer functions for water retention," Soil Science Society of America Journal, vol. 67, no. 4, pp. 1085-1092, 2003.

[5] B. Minasny, J. W. Hopmans, T. Harter, S. O. Eching, A. Tuli, and M. A. Denton, "Neural networks prediction of soil hydraulic functions for alluvial soils using multistep outflow data," Soil Science Society of America Journal, vol. 68, no. 2, pp. 417-429, 2004.

[6] Y. A. Pachepsky, W. J. Rawls, and H. S. Lin, "Hydropedology and pedotransfer functions," Geoderma, vol. 131, no. 3-4, pp. 308316, 2006.

[7] J. H. M. Wösten, P. A. Finke, and M. J. W. Jansen, “Comparison of class and continuous pedotransfer functions to generate soil hydraulic characteristics," Geoderma, vol. 66, no. 3-4, pp. 227237, 1995.

[8] J. Bouma and J. A. van Lanen, "Transfer functions and threshold values: from soil characteristics to land qualities," in Proceedings of the International Workshop on Quantifi ed Land Evaluation Procedures, K. J. Beek, P. A. Burrough, and D. E. McCormack, Eds., pp. 106-111, International Inst. For Aerospace Survey and Earth Sciences, Washington, DC, USA, May 1987.

[9] H. S. Lin, K. J. McInnes, L. P. Wilding, and C. T. Hallmark, "Effects of soil morphology on hydraulic properties. II. Hydraulic pedotransfer functions," Soil Science Society of America Journal, vol. 63, no. 4, pp. 955-961, 1999.
[10] T. Mayr and N. J. Jarvis, "Pedotransfer functions to estimate soil water retention parameters for a modified Brooks-Corey type model," Geoderma, vol. 91, no. 1-2, pp. 1-9, 1999.

[11] B. Minasny and A. B. McBratney, "The neuro-m method for fitting neural network parametric pedotransfer functions," Soil Science Society of America Journal, vol. 66, no. 2, pp. 352-361, 2002.

[12] L. Baker and D. Ellison, "Optimisation of pedotransfer functions using an artificial neural network ensemble method," Geoderma, vol. 144, no. 1-2, pp. 212-224, 2008.

[13] E. Z. Panagou, V. Kodogiannis, and G. J.-E. Nychas, "Modelling fungal growth using radial basis function neural networks: the case of the ascomycetous fungus Monascus ruber van Tieghem," International Journal of Food Microbiology, vol. 117, no. 3, pp. 276-286, 2007.

[14] J. S. R. Jang, "ANFIS: adaptive-network-based fuzzy inference system," IEEE Transactions on Systems, Man and Cybernetics, vol. 23, no. 3, pp. 665-685, 1993.

[15] H. R. Motaghian and J. Mohammadi, "Predictive infiltration rate mapping with improved soil and terrain predictors," Journal of Applied Sciences, vol. 9, no. 8, pp. 1562-1567, 2009.

[16] J. Tomasella, M. G. Hodnett, and L. Rossato, "Pedotransfer functions for the estimation of soil water retention in Brazilian soils," Soil Science Society of America Journal, vol. 64, no. 1, pp. 327338, 2000.

[17] J. H. M. Wösten, Y. A. Pachepsky, and W. J. Rawls, “Pedotransfer functions: bridging the gap between available basic soil data and missing soil hydraulic characteristics," Journal of Hydrology, vol. 251, no. 3-4, pp. 123-150, 2001.

[18] R. Wieland and W. Mirschel, "Adaptive fuzzy modeling versus artificial neural networks," Environmental Modelling and Software, vol. 23, no. 2, pp. 215-224, 2008.

[19] K. Parasuraman, A. Elshorbagy, and B. C. Si, "Estimating saturated hydraulic conductivity in spatially variable fields using neural network ensembles," Soil Science Society of America Journal, vol. 70, no. 6, pp. 1851-1859, 2006.

[20] E. Avci and Z. H. Akpolat, "Speech recognition using a wavelet packet adaptive network based fuzzy inference system," Expert Systems with Applications, vol. 31, no. 3, pp. 495-503, 2006.

[21] J. Ryoo, Z. Dragojlovic, and D. A. Kaminski, "Control of convergence in a computational fluid dynamics simulation using ANFIS," IEEE Transactions on Fuzzy Systems, vol. 13, no. 1, pp. 42-47, 2005.

[22] A. Baylar, D. Hanbay, and E. Ozpolat, "Modeling aeration efficiency of stepped cascades by using ANFIS," Clean-Soil, Air, Water, vol. 35, no. 2, pp. 186-192, 2007.

[23] J. S. Jang and N. Gulley, Fuzzy Logic Toolbox: Reference Manual, The Mathworks, Natick, Mass, USA, 1996.

[24] M. H. Mahdian and R. S. Oskoee, "Developing pedotransfer functions to predict infiltration rate in flood spreading stations of Iran," Research Journal of Environmental Sciences, vol. 3, no. 6, pp. 697-704, 2009.

[25] H. P. Ritzema, Drainage Principles and Applications, ILRI, The Netherlands, 1994.

[26] W. A. Agyare, S. J. Park, and P. L. G. Vlek, "Artificial neural network estimation of saturated hydraulic conductivity," Vadose Zone Journal, vol. 6, no. 2, pp. 423-431, 2007.

[27] K. C. Luk, J. E. Ball, and A. Sharma, "A study of optimal model lag and spatial inputs to artificial neural network for rainfall forecasting," Journal of Hydrology, vol. 227, no. 1-4, pp. 56-65, 2000. 
[28] S. Tamari, J. H. M. Wösten, and J. C. Ruiz-Suárez, “Testing an artificial neural network for predicting soil hydraulic conductivity," Soil Science Society of America Journal, vol. 60, no. 6, pp. 1732-1741, 1996.

[29] I. Yilmaz, M. Marschalko, M. Bednarik, O. Kaynar, and L. Fojtova, "Neural computing models for prediction of permeability coefficient of coarse-grained soils," Neural Computing and Applications, vol. 21, no. 5, pp. 957-968, 2012.

[30] M. G. Schaap and F. J. Leij, "Using neural networks to predict soil water retention and soil hydraulic conductivity", Soil and Tillage Research, vol. 47, no. 1-2, pp. 37-42, 1998.

[31] Y. A. Pachepsky, D. Timlin, and G. Varallyay, "Artificial neural networks to estimate soil water retention from easily measurable data," Soil Science Society of America Journal, vol. 60, no. 3, pp. 727-733, 1996.

[32] M. Kim and J. E. Gilley, "Artificial Neural Network estimation of soil erosion and nutrient concentrations in runoff from land application areas," Computers and Electronics in Agriculture, vol. 64, no. 2, pp. 268-275, 2008.

[33] I. Yilmaz, M. Marschalko, M. Bednarik, O. Kaynar, and L. Fojtova, "Neural computing models for prediction of permeability coefficient of coarse-grained soils," Neural Computing and Applications, pp. 1-12, 2011.

[34] M. Aqil, I. Kita, A. Yano, and S. Nishiyama, "A comparative study of artificial neural networks and neuro-fuzzy in continuous modeling of the daily and hourly behaviour of runoff," Journal of Hydrology, vol. 337, no. 1-2, pp. 22-34, 2007.

[35] H. M. Ertunc and M. Hosoz, "Comparative analysis of an evaporative condenser using artificial neural network and adaptive neuro-fuzzy inference system," International Journal of Refrigeration, vol. 31, no. 8, pp. 1426-1436, 2008.

[36] I. Yilmaz and O. Kaynar, "Multiple regression, ANN (RBF, MLP) and ANFIS models for prediction of swell potential of clayey soils," Expert Systems with Applications, vol. 38, no. 5, pp. 5958-5966, 2011.

[37] T. Takagi and M. Sugeno, "Fuzzy identification of systems and its applications to modeling and control," IEEE Transactions on Systems, Man and Cybernetics, vol. 15, no. 1, pp. 116-132, 1985. 

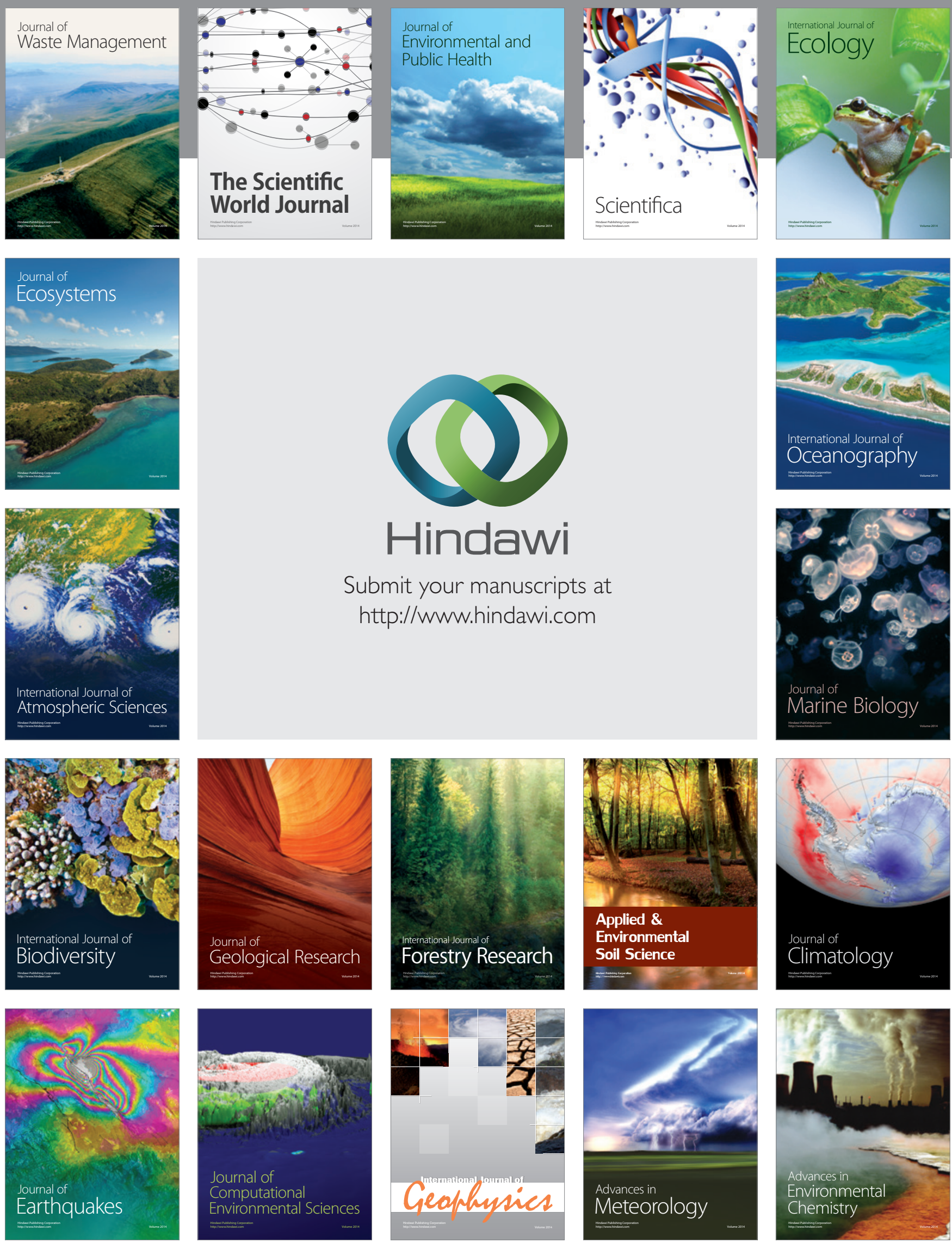